\title{
Interactive comment on "Comparison of CMIP6 Historical Climate Simulations and Future Projected Warming to an Empirical Model of Global Climate” by Laura A. McBride et al.
}

\section{Anonymous Referee \#2}

Received and published: 10 October 2020

This manuscript used a multiple linear regression energy balance model, EM-GC, to estimate the attributable anthropogenic warming rate (AAWR), the equilibrium climate sensitivity (ECS), and the future projections. The authors compared the results from EM-GC with those obtained from CMIP6. They found that the CMIP6 GCMs tend to exhibit a faster rate of warming, which induced larger AAWR, larger ECS, and smaller remaining budgets of carbon emissions. One highlight of this work is the use of Aerosol Weighting Method, which allowed a probabilistic estimation. This work is very interesting and the authors have done many detailed analyses. However, before I can recommend accepting this manuscript, I have several concerns that need to be addressed. 
1. To run the EM-GC model, it seems that one needs to determine nine regression coefficients and parameters. Constrained by the observed GMST and the OHC, one ESDD can obtain a set of the nine coefficients/parameters to ensure a good fit to the historical observations. However, I am not sure if the selected set of coefficients/parameters is unique, or one can use a totally different set of coefficients/parameters to achieve a similar fitting skill? I also have concerns that whether the coefficients/parameters are still useful for the future projections? I would like to suggest the author to perform a test to prove the validity of the model and the stability of the coefficients/parameters. For example, the authors may consider to divide the historical period into two halves, use the first half to determine the coefficients/parameters, and use the second half to test the stability.

2. From Fig. 1f, the authors found that the PDO has very limited contributions to the GMST. I don't understand this finding, as to my knowledge, the different phases of the PDO play an important role in modulating the GMST. For example, the recently well discussed warming hiatus in the beginning of this century has been found to be closely related to the PDO. An explanation about the findings in Fig. $1 \mathrm{f}$ is needed.

3. Another concern is about the comparison of the AAWR that obtained from EM-GC and CMIP6 models. Since different methods are used to calculate the AAWR, I am not sure if the results are comparable. Especially for the CMIP6 models, the REG method seems to be too simple to calculate the AAWR. I am not sure if the AAWR values obtained from the CMIP6 models are as pure as those obtained from EM-GC.

4. In line 228, “. . . also specified on Fig. 1f”, "Fig. 1f” should be "Fig. 1e".

5. In line 975, "then" should be "than".

6. In line 1061, "... of the Paris Agreement will be achieved", "will be" should be "will not be".

Printer-friendly version

Discussion paper

Interactive comment on Earth Syst. Dynam. Discuss., https://doi.org/10.5194/esd-2020-67,

Interactive

comment 
Interactive comment 